Revista Calidad en la Educación Superior

Programa de Autoevaluación Académica

Universidad Estatal a Distancia, Costa Rica

ISSN 1659-4703

revistacalidad@uned.ac.cr

\title{
¿CÓMO EVALUAR EL IMPACTO SOCIAL DE LA FORMACIÓN CONTINUA EN EL CONTEXTO Y ALCANCE DE LA UNIVERSIDAD EN CIENCIAS PEDAGÓGICAS “JOSÉ MARTÍ” DE CAMAGÜEY? RESULTADOS DEL 2012
} AL 2014

HOW TO ASSESS THE SOCIAL IMPACT OF CONTINUING TRAINING IN THE CONTEXT AND SCOPE OF PEDAGOGICAL SCIENCES UNIVERSITY "JOSÉ MARTÍ" CAMAGÜEY? RESULTS OF THE YEARS FROM 2012 TO 2014

Erenia de la C. Martínez Escoda, emartinez@ucp.cm.rimed.cu' ${ }^{1}$
Javier López Fernández, jlopez@ucp.cm.rimed.cu
Belkis Yaisy Zulueta Morciego, bzulueta@ucp.cm.rimed.cu
Universidad de Camagüey "Ignacio Agramonte", Cuba

Volumen 6, número 1

Mayo 2015

Pp. $19-43$

Recibido: 15 de setiembre, 2014

Aprobado: 10 de marzo, 2015

\footnotetext{
1 Erenia Martínez Escoda, Profesor Auxiliar, Doctora en Ciencias Pedagógicas. Directora e investigadora del Centro de Estudios para la Calidad Educacional y Empresarial de la Universidad de Camagüey «Ignacio Agramonte» en Cuba. Correo electrónico: erenia.martinez@reduc.edu.cu

2 Javier López Fernández, Profesor Auxiliar, Máster en Investigación Educativa y profesor investigador del Centro de Estudios para la Calidad Educacional y Empresarial de la Universidad de Camagüey «Ignacio Agramonte» en Cuba. Correo electrónico: javier.lopez@reduc.edu.cu

${ }^{3}$ Belkis Yaisy Zulueta Morciego, Asistente, Máster en Educación Superior y profesor investigador del Centro de Estudios para la Calidad Educacional y Empresarial de la Universidad de Camagüey «lgnacio Agramonte» en Cuba. Correo electrónico: belkis.zulueta@reduc.edu.cu
} 


\section{Resumen}

El artículo describe la metodología general para la evaluación del impacto social de la formación continua en el contexto y alcance de la Universidad en Ciencias Pedagógicas "José Martí" en Cuba, así como los resultados y las experiencias alcanzados desde el año 2012 hasta el 2014 por el Centro de Estudios para la Evaluación de la Calidad Educacional. Se emplean métodos en los niveles teórico, empírico y estadístico, y como resultado se determinan los impactos sociales, partiendo de la sistematización de contenidos relativos a la calidad en la educación superior pedagógica, asociada a la obtención de la excelencia académica en relación con la pertinencia social. Además, sistematiza el tránsito desde un proceso macroevaluativo que brinda resultados iniciales hasta subprocesos microevaluativos que contienen estudios de profundización.

Palabras claves: diversidad, calidad, impacto social, evaluación de impacto social

\section{Abstract}

The paper describes the general methodology for evaluating the social impact of continuing training in the context and scope of Pedagogical Sciences University "José Martí" in Cuba, as well as results and experiences gained from 2012 to 2014 by the Center Study for the Evaluation of Educational Quality. Methods of theoretical, empirical and statistical is empelaron. As a result, social impacts, based on the systematization of content relating to quality in teaching higher education, associated with the procurement of academic excellence for social relevance, besides traffic from systematized process that provides macroevaluativo obtained initial results until microevaluativos threads containing depth studies.

Keywords: diversity, quality, social impact and social impact assessment.

Las actuales circunstancias en las que se desarrolla la educación superior pedagógica en Cuba están permeadas por transformaciones sustanciales y dinámicas vistas desde códigos dialécticos materialistas de un profundo carácter humanista, estimuladas, en lo esencial, por exigencias que devienen de una realidad inmersa en las transformaciones económicas y sociales que visualizan al hombre y a la mujer como elementos catalizadores del cambio. (Espí., N. 2012, p.4) 
En conjunto con estas transformaciones, los procesos de acreditación y/o certificación -inmersos en la búsqueda de instituciones de excelencia académica, en unidad con la pertinencia social — juegan un rol fundamental, por lo que son un punto de partida para la evaluación del impacto social, centrado en la misión de lograr las aspiraciones que se reflejan en los patrones de calidad para los procesos sustantivos que se gestan y enriquecen la formación de docentes para los subsistemas educacionales.

Para alcanzar la excelencia académica, el impacto social es uno de los objetivos esenciales que permiten investigar mediante la evaluación de niveles de satisfacción en los órdenes personal, organizacional y social. Es así que surge la siguiente pregunta: ¿Cuál es el resultado inmediato de las trascendencias del proceso de formación continua con alcance a los profesionales pedagógicos?, con el fin de poner en relieve los efectos múltiples sobre el entorno social.

Lo anteriormente expuesto y la aspiración de cumplir con el objetivo que el Ministerio de Educación, en su labor sistemática por mejorar el proceso de formación de docentes, estableció —en el cual se declara que es fundamental "priorizar la formación inicial y permanente de los profesionales de la Educación, perfeccionando la concepción de la universalización de la Educación Superior, con énfasis en la superación sistemática del docente, para garantizar la calidad del proceso docente y educativo" (MINED, 2008, p.2)—convierten en vital la necesidad del monitoreo sistemático de la calidad educacional y, con ello, del impacto social en los procesos universitarios.

En este sentido, se les encomendó a las personas investigadoras del Centro de Estudios para la Evaluación de la Calidad Educativa de la Universidad en Ciencias Pedagógicas "José Marti" de Camagüey la tarea de evaluar el impacto social en los procesos de formación continua, para lo cual se elaboró una metodología. 
El objetivo de este artículo es describir la metodología general para la evaluación del impacto social de la formación continua en el contexto y alcance de la Universidad en Ciencias Pedagógicas "José Martí" en Cuba, así como los resultados y las experiencias alcanzados del 2012 al 2014 por el Centro de Estudios para la Evaluación de la Calidad Educacional en este proceder.

Desde la perspectiva dialéctica materialista asumida se acometió una sistematización de los referentes en torno a la evaluación de la calidad educacional en Cuba, de la evaluación del impacto social y de su relación con el nivel de satisfacción en el entorno social. Esta sistematización permitió precisar los fundamentos de la metodología general para la evaluación del impacto social de la formación continua en el contexto y alcance de la Universidad en Ciencias Pedagógicas “José Martí” y sus respectivos métodos y procedimientos.

La modelación fue empleada para describir el proceso y precisar los ciclos correspondientes de la metodología general para la evaluación del impacto social de la formación continua y las acciones que se desarrollaron.

En la investigación de la que da cuenta este artículo se empleó, además, el experimento pedagógico, a fin de confirmar la efectividad de la propuesta y obtener los resultados esperados que se describen en el texto.

\section{Resultados y discusión}

El estudio realizado permitió confirmar que la metodología para la evaluación del impacto social de la formación continua en el contexto y alcance de la Universidad en Ciencias Pedagógicas "José Marti”" cuenta con un ciclo de vida (Martínez, E.; López, J., Pozas, WJ. y Marrero, O., 2013, p.8) 


\section{Figura 1. Representación del ciclo de vida de la metodología general para la evaluación del impacto social}

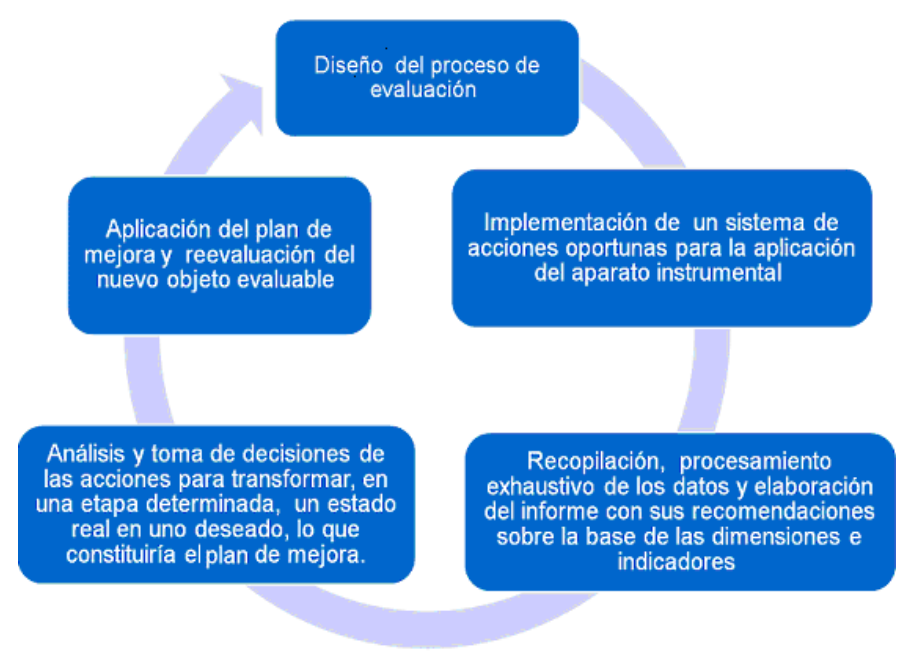

Fuente: Elaboración propia.

Un análisis de las definiciones conceptuales relacionadas con el tema permitió definir la evaluación del impacto social de la formación continua en el contexto y alcance de la UCP "José Marti" como el proceso de recopilación de información válida y confiable que permite establecer niveles de satisfacción en los órdenes personal, organizacional y social, sobre las trascendencias en el contexto y alcance de esta institución en cuanto al proceso de formación continua, con el objetivo de promover acciones de mejora que puedan dar lugar al alcance de la excelencia académica de acuerdo a los patrones de calidad establecidos para este proceso, sin excluir la importancia de las funciones de diagnóstico y de valoración, necesarias para obtener la finalidad con cierto nivel de efectividad (Martínez, E.; López, J., Pozas, WJ. y Marrero, O., 2013, p.10) .

En la metodología general, para evaluar el impacto social de la formación continua en la primera fase, la etapa de diseño asumió que la variable de evaluación es el 
impacto social de la formación continua en el contexto y alcance de la UCP "José Martí".

Por la complejidad de esta variable, para obtener información fidedigna se realizó su operacionalización, con lo cual se obtuvieron como dimensiones el impacto social de los procesos de formación de pregrado y postgrado y el resultado de las investigaciones.

La operacionalización realizada se justifica, pues una de las variables incluidas en el patrón de calidad elaborado por el Ministerio de Educación Superior (MES) lo constituye: el impacto social de los procesos sustantivos de las universidades, entre los que se encuentran los ya declarados en esta investigación.

La primera dimensión tiene que ver con el impacto social de la formación inicial en el contexto y alcance de la UCP "José Martí" y comprende entre sus subdimensiones los niveles de satisfacción en cuanto al proceso de la formación inicial de educadores y educadoras, la satisfacción vinculada a la formación profesional inicial alcanzada por la persona egresada y, finalmente, su contribución al desarrollo social de la comunidad (Martínez, E.; López, J., Pozas, WJ. y Marrero, O., 2013, p.11)

La segunda dimensión profundiza en el impacto social de la formación postgraduada en el contexto y alcance de la UCP "José Martí" y comprende entre sus subdimensiones los niveles de satisfacción en cuanto al proceso de formación postgraduada, la satisfacción vinculada al crecimiento profesional operado y la contribución de las personas egresadas al desarrollo educativo de la comunidad (Martínez, E.; López, J., Pozas, WJ. y Marrero, O., 2013, p.11)

La última dimensión se relaciona con el impacto social de los resultados de las investigaciones en el contexto y alcance de la UCP "José Martí" e incluye entre 
sus subdimensiones los niveles de satisfacción vinculados al desarrollo de las investigaciones de las personas educadoras, la introducción de sus resultados y la contribución a la solución de los problemas educativos de la comunidad (Martínez, E.; López, J., Pozas, WJ. y Marrero, O., 2013, p.11)

Luego de un estudio sistemático de cada una de las dimensiones y subdimensiones que demuestran la diversidad de factores que implican constantes efectos múltiples sobre el objeto evaluable, se determinaron los indicadores teniendo como base los estándares del patrón de calidad y, a su vez, se elaboraron los instrumentos (Martínez, E.; López, J., Pozas, WJ. y Marrero, O., 2013, p.111)

Las encuestas son diversas en su contenido, en primer lugar porque cada una está destinada a diferentes clases de la muestra seleccionada, las cuales emiten criterios múltiples según los juicios valorativos que cada una tiene e indican un nivel de satisfacción determinado sobre el objeto evaluable (Martínez, E.; López, J., Pozas, WJ. y Marrero, O., 2013, p.13)

En segundo lugar, los ítems de cada encuesta se relacionan con indicadores de diferentes subdimensiones y dimensiones que reflejan exhaustivamente la vida académica intra y extra universitaria. De igual manera, se aplicaron otras técnicas, como las entrevistas semiestructuradas a profundidad y el muestreo de documentos.

Esta concepción, dirigida a triangular las relaciones que se establecen en el proceso investigativo, permite una mejor aproximación a la realidad estudiada. Al respecto, Rodríguez, Gil y García señalaron lo siguiente:

Su empleo permite realizar la triangulación en cada uno de los indicadores y obtener una aproximación de la realidad estudiada, 
estando seguros que si diversas clases de datos conducen a la misma conclusión podemos estar algo más seguros de tal conclusión (Rodríguez, Gil y García, 2002, p.287).

Por consiguiente, para cada una de las dimensiones y subdimensiones se asumieron los siguientes indicadores:

\section{Tabla 1. Operacionalización de la variable de la investigación en dimensiones, subdimensiones e indicadores}

\begin{tabular}{|c|c|c|}
\hline Dimensiones & Subdimensiones & Indicadores \\
\hline \multirow{8}{*}{$\begin{array}{l}\text { 1. Impacto } \\
\text { socioeducativo } \\
\text { en la formación } \\
\text { inicial de las } \\
\text { futuras } \\
\text { personas } \\
\text { egresadas }\end{array}$} & \multirow{6}{*}{$\begin{array}{lr}\text { 1.1. Satisfacción en } \\
\text { cuando r al } \\
\text { proceso de } \\
\text { formación inicial }\end{array}$} & $\begin{array}{l}\text { 1.1.1. Nivel de satisfacción en cuanto } \\
\text { al plan de estudio de la carrera }\end{array}$ \\
\hline & & $\begin{array}{l}\text { 1.1.2. Nivel de satisfacción vinculado } \\
\text { al funcionamiento de los } \\
\text { colectivos de carrera, año y } \\
\text { disciplina }\end{array}$ \\
\hline & & $\begin{array}{l}\text { 1.1.3. Nivel de satisfacción } \\
\text { relacionado con la calidad de la } \\
\text { formación académica de la } \\
\text { población estudiantil y la de los y } \\
\text { las docentes en formación }\end{array}$ \\
\hline & & $\begin{array}{l}\text { 1.1.4. Nivel de satisfacción en cuanto } \\
\text { a la calidad del proceso de } \\
\text { formación laboral de la } \\
\text { población estudiantil y de los y } \\
\text { las docentes en formación }\end{array}$ \\
\hline & & $\begin{array}{l}\text { 1.1.5. Nivel de } \\
\text { relacionado con el proceso de } \\
\text { formación para el trabajo } \\
\text { investigativo }\end{array}$ \\
\hline & & $\begin{array}{l}\text { 1.1.6. Nivel de satisfacción vinculado } \\
\text { al proceso de formación } \\
\text { ideopolítica de los y las } \\
\text { estudiantes }\end{array}$ \\
\hline & \multirow{2}{*}{$\begin{array}{l}\text { 1.2. Satisfacción en } \\
\text { cuanto a la } \\
\text { formación } \\
\text { profesional }\end{array}$} & $\begin{array}{l}\text { 1.2.1. Nivel de satisfacción en cuanto } \\
\text { al dominio del contenido }\end{array}$ \\
\hline & & $\begin{array}{l}\text { 1.2.2. Nivel de satisfacción vinculado } \\
\text { a la preparación profesional }\end{array}$ \\
\hline
\end{tabular}




\begin{tabular}{|c|c|c|}
\hline Dimensiones & Subdimensiones & Indicadores \\
\hline & \multirow[t]{4}{*}{ inicial } & $\begin{array}{l}\text { 1.2.3. Nivel de satisfacción } \\
\text { relacionado con la calidad de la } \\
\text { educación que les ofrece a sus } \\
\text { estudiantes. }\end{array}$ \\
\hline & & $\begin{array}{l}\text { 1.2.4. Nivel de satisfacción en cuanto } \\
\text { a la integración a las tareas } \\
\text { sociales }\end{array}$ \\
\hline & & $\begin{array}{l}\text { 1.2.5. Nivel de satisfacción vinculado } \\
\text { a la conducta que mantiene en } \\
\text { la comunidad. }\end{array}$ \\
\hline & & $\begin{array}{l}\text { 1.2.6. Nivel de satisfacción } \\
\text { relacionado con el trabajo de } \\
\text { formación de valores con sus } \\
\text { estudiantes. }\end{array}$ \\
\hline & \multirow{4}{*}{$\begin{array}{l}\text { 1.3. Contribución de } \\
\text { la persona } \\
\text { egresada al } \\
\text { desarrollo } \\
\text { educativo de la } \\
\text { comunidad }\end{array}$} & $\begin{array}{l}\text { 1.3.1. Nivel de cobertura laboral } \\
\text { profesional de cada una de las } \\
\text { educaciones }\end{array}$ \\
\hline & & $\begin{array}{l}\text { 1.3.2. Media de los resultados de los } \\
\text { indicadores de eficiencia por } \\
\text { educaciones en los últimos cinco } \\
\text { años }\end{array}$ \\
\hline & & $\begin{array}{l}\text { 1.3.3. Realización de actividades } \\
\text { comunitarias }\end{array}$ \\
\hline & & $\begin{array}{l}\text { 1.3.4. Lugar alcanzado por las } \\
\text { educaciones de la provincia en } \\
\text { los resultados de los principales } \\
\text { indicadores en los últimos cinco } \\
\text { años }\end{array}$ \\
\hline \multirow{4}{*}{$\begin{array}{l}\text { 2. Impacto } \\
\text { socioeducativo } \\
\text { de la formación } \\
\text { postgraduada }\end{array}$} & \multirow{4}{*}{$\begin{array}{l}\text { 2.1. Satisfacción en } \\
\text { cuanto al al } \\
\text { proceso de } \\
\text { formación } \\
\text { postgraduada }\end{array}$} & $\begin{array}{l}\text { 2.1.1. Nivel de satisfacción vinculado } \\
\text { a la pertinencia }\end{array}$ \\
\hline & & $\begin{array}{l}\text { 2.1.2. Nivel de satisfacción en cuanto } \\
\text { a la calidad de los cursos } \\
\text { (Comprende actualidad del } \\
\text { contenido, gestión de la } \\
\text { información y uso de las nuevas } \\
\text { tecnologías.) }\end{array}$ \\
\hline & & $\begin{array}{l}\text { 2.1.3. Nivel de satisfacción } \\
\text { relacionado con la accesibilidad }\end{array}$ \\
\hline & & $\begin{array}{l}\text { 2.1.4. Nivel de satisfacción vinculado } \\
\text { a la preparación profesional de } \\
\text { los y las docentes que } \\
\text { desarrollan los cursos. }\end{array}$ \\
\hline
\end{tabular}




\begin{tabular}{|c|c|c|}
\hline \multirow{8}{*}{ Dimensiones } & Subdimensiones & Indicadores \\
\hline & \multirow{5}{*}{$\begin{array}{l}\text { 2.2. Satisfacción } \\
\text { con relación al } \\
\text { crecimiento } \\
\text { profesional } \\
\text { operado }\end{array}$} & $\begin{array}{l}\text { 2.2.1. Nivel de satisfacción en cuanto } \\
\text { al dominio del contenido de la } \\
\text { especialidad recibido }\end{array}$ \\
\hline & & $\begin{array}{l}\text { 2.2.2. Nivel de satisfacción } \\
\text { relacionado con la calidad de las } \\
\text { actividades docentes } \\
\text { metodológicas que desarrolla. }\end{array}$ \\
\hline & & $\begin{array}{l}\text { 2.2.3. Nivel de satisfacción vinculado } \\
\text { a la calidad de las actividades } \\
\text { educativas de la persona } \\
\text { egresada }\end{array}$ \\
\hline & & $\begin{array}{l}\text { 2.2.4. Nivel de satisfacción en cuanto } \\
\text { a la calidad de la labor } \\
\text { extensionista universitaria que } \\
\text { realiza. }\end{array}$ \\
\hline & & $\begin{array}{l}\text { 2.2.5. Nivel de satisfacción } \\
\text { relacionado con la calidad de la } \\
\text { producción intelectual, que debe } \\
\text { transformar, proteger y dignificar } \\
\text { los valores naturales, culturales } \\
\text { o históricos de la escuela y la } \\
\text { comunidad. }\end{array}$ \\
\hline & \multirow{2}{*}{$\begin{array}{l}\text { 2.3. Contribución de } \\
\text { la persona } \\
\text { egresada al } \\
\text { desarrollo } \\
\text { socioeducativo } \\
\text { de la } \\
\text { comunidad }\end{array}$} & $\begin{array}{l}\text { 2.3.1. Organización de actividades } \\
\text { tendientes al desarrollo } \\
\text { sociocultural y político-ideológico } \\
\text { de la comunidad como resultado } \\
\text { de su formación postgraduada }\end{array}$ \\
\hline & & $\begin{array}{l}\text { 2.3.2. Mejoras en el trabajo educativo } \\
\text { de la escuela como resultado de } \\
\text { la aplicación de los estudios } \\
\text { posgraduados realizados }\end{array}$ \\
\hline \multirow{5}{*}{$\begin{array}{l}\text { 3. Impacto } \\
\text { socioeducativo } \\
\text { de los resultados } \\
\text { de las } \\
\text { investigaciones }\end{array}$} & \multirow{3}{*}{$\begin{array}{l}\text { 3.1. Satisfacción en } \\
\text { cuanto } \\
\text { desarrollo al } \\
\text { las } \\
\text { investigaciones }\end{array}$} & $\begin{array}{l}\text { 3.1.1. Ajuste del desarrollo de la } \\
\text { investigación al cronograma } \\
\text { elaborado }\end{array}$ \\
\hline & & 3.1.2. Pertinencia de la investigación \\
\hline & & $\begin{array}{l}\text { 3.1.3. Obtención de resultados } \\
\text { previstos y no previstos }\end{array}$ \\
\hline & \multirow{2}{*}{$\begin{array}{l}\text { 3.2. Satisfacción } \\
\text { con relación a } \\
\text { la introducción } \\
\text { de los } \\
\text { resultados }\end{array}$} & $\begin{array}{l}\text { 3.2.1. Divulgación de los resultados } \\
\text { 3.2.2. Socialización de los resultados }\end{array}$ \\
\hline & & $\begin{array}{l}\text { 3.2.3. Desarrollo de cursos de } \\
\text { postgrado a partir de los } \\
\text { resultados de la investigación }\end{array}$ \\
\hline
\end{tabular}




\begin{tabular}{|c|c|c|}
\hline Dimensiones & Subdimensiones & Indicadores \\
\hline & $\begin{array}{c}\text { 3.3. Contribución a } \\
\text { la solución de } \\
\text { los problemas } \\
\text { educativos de la } \\
\text { comunidad }\end{array}$ & $\begin{array}{c}\text { 3.3.1. Contribución de los resultados } \\
\text { a la solución de los problemas } \\
\text { de la comunidad o de la escuela }\end{array}$ \\
\cline { 3 - 3 } & $\begin{array}{c}\text { 3.3.2. Transformaciones logradas en } \\
\text { los lugares donde se aplicaron } \\
\text { los resultados. }\end{array}$ \\
\hline
\end{tabular}

Fuente: Recuperado de Martínez, E. y López, J. (2012)

Una muestra más detallada de los instrumentos elaborados durante el primer ciclo de la metodología (diseño del proceso de evaluación), empleados en la evaluación del impacto social de la formación continua en el contexto y alcance de la UCP "José Martí" de la provincia de Camagüey en Cuba, explicita la diversidad de la muestra según su implicación en el proceso, en la que se aplicaron los instrumentos de investigación necesarios y suficientes para obtener información válida, relacionados a continuación:

- Encuesta a docentes de la UCP “José Marti” (E1). Se les aplicó a los y las docentes que trabajan en una carrera.

- Encuesta a estudiantes de la UCP "José Martí" (E2). Se aplicó en dependencia de las matrículas de las carreras; se propone una muestra representativa.

- Encuesta a personas egresadas de la UCP "José Martí" (E3). Se aplicó una muestra con el mayor nivel de representatividad.

- Encuesta a tutores y tutoras de las instituciones educativas (E4). Se les aplicó a los tutores y las tutoras de estudiantes y de la población egresada que integra la muestra.

- Encuesta a la familia (E5). Se aplicó a una muestra de familiares de los y las estudiantes y de la población docente en formación o egresada.

- Encuesta a los y las líderes de la comunidad (E6). Se les aplicó a los y las líderes de organizaciones sociales y del Gobierno de la localidad donde vive la persona docente en formación o la egresada. 
- Encuesta a empleadores y empleadoras (E7). Se les aplicó a los directores $\mathrm{y}$ las directoras de las instituciones educativas, jefes $\mathrm{y}$ jefas de departamentos y metodólogos y metodólogas municipales que atienden las asignaturas en las que trabaja la persona docente en formación o la egresada.

- Entrevista a directivos y directivas de educación (E8). Se les aplicó a los directivos y las directivas de los subsistemas de educación donde se desempeña la población docente en formación y la egresada.

- Entrevista semiestructurada a profundidad: Se les aplicó a tutores y tutoras, directivos y directivas y familiares de las personas docentes en formación o las egresadas.

Se realizó un muestreo de documentos a estrategia de superación, programas, planes de clases $u$ otras formas organizativas, libretas de estudiantes, tesis de diplomas, maestrías y doctorados, resultados de proyectos de investigación, expedientes escolares y documentos de la DICP, Dirección de Información Científico-Pedagógica (Martínez, E.; López, J., Pozas, WJ. y Marrero, O., 2013, p.111)

Así por ejemplo, uno de los instrumentos elaborados se relaciona con la encuesta aplicada a los y las líderes de las comunidades; este instrumento abarcó una gama amplia de indicadores y a su vez evaluó el grado de satisfacción que sienten los y las líderes en cuanto al desempeño de la población docente, vista desde la extensión de su aporte a la comunidad de residencia, aspecto trascendental que va a permitir implantar planes de mejora, teniendo en cuenta la satisfacción vinculada a la calidad de la educación de la población que compone el entorno social (Martínez, E.; López, J., Pozas, WJ. y Marrero, O., 2013, p.61) 
A su vez, en las encuestas aplicadas a la población docente, a los empleadores y las empleadoras de dicha población y a las y los dirigentes se recogieron datos que permiten apreciar el proceso de forma inversa, o sea, el grado de satisfacción que sienten los y las docentes en cuanto a los y las líderes de la comunidad, vistos desde las relaciones de trabajo educacional que necesariamente se establecen en la formación de docentes en el entorno social.

Para ejemplificar, a continuación se muestra la encuesta realizada a los y las líderes:

Estimado compañero, desde su condición de líder comunitario requerimos que realice algunas valoraciones acerca de los maestros y profesores que viven o desarrollan su labor docente en la comunidad que usted representa, ello tiene como finalidad mejorar la calidad de la educación que se imparte en la Universidad de Ciencias Pedagógicas "José Martí", por tanto, le solicitamos que responda con sinceridad. De antemano agradecemos su contribución. 
Marque con una $X$ según considere.

Organización social que Ud. representa en la comunidad:

\begin{tabular}{|l|l|l|l|l|l|}
\hline $\begin{array}{l}\text { La mayoría de los maestros y profesores que viven en } \\
\text { su comunidad. }\end{array}$ & No & $\begin{array}{l}\text { A } \\
\text { veces }\end{array}$ & $\begin{array}{l}\text { No } \\
\text { sé }\end{array}$ & $\begin{array}{l}\text { No } \\
\text { procede }\end{array}$ \\
\hline $\begin{array}{l}\text { 1. Apoyan las tareas orientadas por las organizaciones } \\
\text { de masas, órganos del poder popular, salud pública, } \\
\text { etc. }\end{array}$ & & & & \\
\hline 2. Por su conducta en general: & & & & \\
\hline $\begin{array}{l}\text { 2.1. Son ejemplo de entrega y sacrificio para los } \\
\text { niños y jóvenes. }\end{array}$ & & & & \\
\hline 2.2. Son respetados por los adultos. & & & & \\
\hline $\begin{array}{l}\text { 2.3. Se han ganado el cariño de los miembros de la } \\
\text { comunidad. }\end{array}$ & & & & \\
\hline $\begin{array}{l}\text { 2.4. Están en capacidad para intervenir en conflictos } \\
\text { y solucionarlos. }\end{array}$ & & & & \\
\hline $\begin{array}{l}\text { 2.5. Tienen capacidad y voluntad para ser líderes } \\
\text { comunitarios. }\end{array}$ & & & & \\
\hline 3. Son cuidadosos con su imagen personal. & & & & \\
\hline 4. Al realizar actividades comunitarias: & & & & \\
\hline $\begin{array}{l}\text { 4.1. Utilizan medios tecnológicos para facilitar las } \\
\text { comunicaciones. }\end{array}$ & & & & \\
\hline $\begin{array}{l}\text { 4.1. Crean o integran grupos para dinamizar el trabajo } \\
\text { comunitario. }\end{array}$ & & & & \\
\hline $\begin{array}{l}\text { 4.2. Organizan concursos en los diferentes grupos } \\
\text { etarios. }\end{array}$ & & & & \\
\hline $\begin{array}{l}\text { 4.3. Las actividades dignifican los valores naturales, } \\
\text { culturales o históricos de la comunidad. }\end{array}$ & & & & \\
\hline $\begin{array}{l}\text { 4.4. Investigan para mitigar los problemas } \\
\text { socioeducativos que aquejan a la comunidad. }\end{array}$ & & & & \\
\hline $\begin{array}{l}\text { 4.5. Intervienen activamente en la protección del medio } \\
\text { ambiente. }\end{array}$ & & & & \\
\hline $\begin{array}{l}\text { 5. Los niños o jóvenes de la comunidad que son sus } \\
\text { estudiantes: }\end{array}$ & & & & \\
\hline 5.1. Aprenden óptimamente. & & & & \\
\hline $\begin{array}{l}\text { 5.2. Mantienen una conducta adecuada en la } \\
\text { comunidad. } \\
\text { conducta de sus hijos. }\end{array}$ & & & & \\
\hline 5.3. Participan en las actividades comunitarias. & & & \\
\hline $\begin{array}{l}\text { Los padres de los niños y jóvenes que son sus } \\
\text { aprendizaje y }\end{array}$ & & & & \\
\hline
\end{tabular}

7. Si desea hacer algún comentario con relación a la calidad en la formación de maestros y profesores por la UCP "José Martí", le agradecemos que lo escriba en este espacio. 
En la encuesta anterior los ítems se corresponden con indicadores de diferentes subdimensiones, el resto de los instrumentos aplicados siguió esta idea y permitió evaluar el impacto social en los procesos sustantivos declarados en la investigación, lo que proporcionó estudios evaluativos de mayor profundidad desde una lógica que implica lo general, lo particular y lo singular e integró toda la diversidad de los procesos desarrollados en la universidad.

Una vez establecido el diseño, la segunda fase comprendió la implementación de un sistema de acciones oportunas para la aplicación de los instrumentos.

El sistema permitió, entre otras cosas, comprender el porqué de la aplicación de los instrumentos y favoreció la formación de convicciones referentes a la investigación, tanto en la persona que aplica y procesa los instrumentos como en la población encuestada, lo cual posibilitó la obtención de mayor confiabilidad de la información a procesar.

Las acciones para esta fase fueron:

- Socializar la metodología con el objetivo de informar su contenido

- Capacitar a los cuadros, jefes y jefas de departamento y personas que aplican los instrumentos de la investigación.

- Estudiar las características psicológicas de la población de la muestra seleccionada

- Estudiar el contexto en el que interactúa la muestra seleccionada.

- Distribuir los instrumentos de acuerdo al número de la muestra representativa

- Crear las condiciones necesarias para la aplicación de los instrumentos (locales y su ambientación, aseguramiento de la asistencia mediante la planificación del ejercicio y de un clima favorable entre los aplicadores y las aplicadoras y las personas que componen la muestra.) 
- Moderar las posturas nocivas respecto a la investigación, tales como el llamado efecto de halo o los errores de severidad y de indulgencia o lenidad.

Para la investigación se asume como efecto de halo el hecho de

Evaluar un objeto guiándose por la impresión general que se tiene del mismo (...) Ejemplos propios de dicho efecto fueron alabar la formación continua porque en ella se han graduado estudiantes de mucho prestigio, defender a los egresados jóvenes y criticar a los más antiguos (...) pensar que un directivo o profesor desarrolla correctamente sus funciones porque nos recibe y trata amablemente (Rodríguez, Gil y García, 2002, pp.159-160).

Por su parte, los errores de severidad y de indulgencia consisten en "conceder puntuaciones bajas a los reactivos", o por el contrario otorgar "puntuaciones demasiado altas" (Rodríguez, Gil y García, 2002, pp.159-160), respectivamente en la escala valorativa empleada.

La tercera fase estableció la recopilación, el procesamiento y la interpretación exhaustiva de los datos y la elaboración del informe con sus recomendaciones, teniendo como pauta la operacionalización de la variable de la investigación. Es en este momento en el que se diseñó y puso en funcionamiento la base de datos en el procesador de cálculo IBM-SPS licitado por la institución evaluadora, de acuerdo a los ítems que guardan relación con los indicadores de las variables.

Las hojas de cálculo se concibieron para registrar la información en correspondencia con una escala valorativa de 1 a 10 , donde 10 es el valor máximo positivo y uno el valor mínimo del efecto del impacto. El procesamiento de la información posibilita calcular un índice para el indicador y para cada persona 
que integra la muestra en un rango amplio de respuestas que, llevado a una escala cualitativa, indica el nivel de satisfacción en cuanto a la actividad evaluada. La escala considera los rangos máximos y mínimos de sus valores, lo que facilita describir en la evaluación no solo el nivel de satisfacción en el que se encuentra el indicador tácito, sino también precisar cualitativamente la categoría en el límite de tolerancia superior o inferior de la escala, denotando así el carácter dialéctico del efecto del impacto y su tendencia. La interpretación de los datos obtenidos se apega a la ética del investigador y a la que promueve la investigación, sin desestimar el grado de experiencia en el proceso que se evaluó, minimizando los errores que por interpretación subjetiva se manifiestan en la investigación.

La escala para la conversión del indicador a valores cualitativos del nivel de satisfacción se presenta a continuación:

Tabla 2. Escala de conversión

\begin{tabular}{|l|l|l|l|}
\hline \multirow{2}{*}{ Categoría } & \multirow{2}{*}{ Simbología } & Escala \\
\cline { 3 - 4 } & & Inf. & Máx. \\
\hline Muy satisfecho & MS & 0,9 & 1 \\
\hline Bastante satisfecho & BS & 0,8 & 0,9 \\
\hline Satisfecho & S & 0,6 & 0,8 \\
\hline Poco satisfecho & PS & 0,4 & 0,6 \\
\hline Insatisfecho & IS & 0 & 0,4 \\
\hline
\end{tabular}

Fuente: Martínez, E.; López, J., Pozas, WJ. y Marrero, O., 2013, p.16).

Otras de las acciones de esta fase fueron:

- Organizar y optimizar la captura de la información en función del tiempo y los recursos humanos

- Validar la información procedente de los instrumentos para evitar que tuviera errores de indulgencia y severidad o que por su grafía ofreciera 
dudas, del mismo modo que la información cuantitativa registrada en la base de datos para evitar omisiones o cambios de códigos.

- Consolidar la información procesada en la base de datos

- Identificar la información susceptible de profundizar para aplicar entrevistas semiestructuradas a profundidad que complementaran el proceso de evaluación en el contexto de la propia investigación.

- Elaborar el informe conclusivo de conjunto con los decisores implicados en la investigación

En la cuarta fase, vinculada al análisis y la toma de decisiones de las acciones, lo esencial fue elaborar el plan de mejoras, para lo cual se hizo necesario tener presente aquellos indicadores que señalan los niveles de satisfacción e insatisfacción, lo que resultó en fortalezas y debilidades.

El plan de mejoras se redactó de forma precisa, conteniendo las acciones a ejecutar, complementadas con la persona responsable, la fecha, los elementos que posibilitan su cumplimiento y las formas de control, además de los datos que indican evidencias. Es importante considerar que son los decisores quienes elaboran este plan de mejoras, en las figuras de los cuadros y de las y los jefes de departamentos docentes y de carreras de la universidad.

La metodología sistematizó los elementos del procedimiento metodológico para la elaboración de un plan de mejoras derivado del proceso de acreditación de universidades (López y García, 2011), el cual, una vez contextualizado, se relacionó con los siguientes objetivos:

1. Extraer cuáles son los señalamientos positivos o negativos con relación a las satisfacciones de las personas encuestadas o entrevistadas.

2. Investigar, mediante la triangulación de los instrumentos utilizados y la revisión documental, los criterios individuales que no son regulares y que es 
necesario analizar según su forma de reflejar los señalamientos, si se relacionan o no con las insatisfacciones y, si no existen en el plan de mejoras, tenerlos en cuenta, pues en todos los casos es posible reflejar la totalidad de los elementos planteados.

3. Tener en cuenta las consideraciones realizadas por las personas expertas en los intercambios $u$ otras valoraciones derivadas de los controles realizados, y que no siempre pasan a formar parte de un informe final en una investigación a cualquier nivel.

4. Reagrupar los señalamientos relacionados con su correspondiente área de resultado clave, de acuerdo a las dimensiones declaradas para la investigación. Téngase en cuenta que los elementos incidentes en los resultados de las diferentes dimensiones evaluadas, en algunos casos, se repiten o aparecen reflejados con diferente lenguaje, grado de importancia o intensidad, debido a que estos reactivos de los instrumentos se derivan de los estándares del patrón de calidad, en los cuales la importancia o intensidad toma matices diferentes.

5. Con la información anterior se sugiere construir una tabla de señalamientos por prioridades y dimensiones, para eliminar las repeticiones y definir cuáles son las más importantes y urgentes prioridades a atender.

6. Derivado de esto, y con la participación de las personas implicadas, diseñar un sistema de acciones de mejora que sea concreto y que tenga en cuenta el estado de los indicadores, las metas a tener en consideración y los plazos planificados para el logro paulatino de ellas, con tareas a desarrollar por una persona en concreto y estableciendo una fecha de cumplimiento.

En la quinta fase fue posible la ejecución del plan de mejora y reevaluación del nuevo objeto evaluable. Para ello fue imprescindible socializar este plan y hacer conscientes a los y las participantes sobre la importancia que reviste el cumplimiento de las acciones, con el único fin de lograr las aspiraciones que, 
como encomienda de la sociedad, tiene la universidad. También es oportuno si es preciso rediseñar las acciones, sobre todo las de control, sabiendo que la práctica es el criterio de la verdad.

En este momento los decisores tenían clara la existencia de impactos inmediatos al aplicar el plan de mejora, por lo que fue posible diseñar nuevamente y comenzar un nuevo ciclo de la evaluación del impacto social en la formación continua, lo que se materializó al comenzar una fase superior del diseño del proceso de evaluación de esta metodología.

Justamente esta práctica evaluativa, que implica un proceso diverso por su naturaleza y su investigación sobre la educación, les ha otorgado a docentes y a directivos y directivas un reflejo más acertado del objeto evaluado.

Por consiguiente, los resultados en el orden teórico-práctico obtenidos por el centro de estudio se unen a la concepción de evaluación de la calidad de los procesos universitarios, vistos como el sistema de aseguramiento y mejoramiento continuo de todas las áreas de la entidad educativa para la formación de profesionales, esto permite la comparación entre los objetivos propuestos y los resultados y propicia la participación activa de todos los factores implicados en el desarrollo de productos y servicios que satisfacen las necesidades de la sociedad, logrando con ello mayor calidad, pertinencia e impacto, con eficiencia, eficacia y efectividad (Addine y García, 2002, p.3).

La medición del impacto es una forma de evaluación que ha ido ganando aceptación entre las personas encargadas de planificar e impartir formación continua; es parte esencial del sistema sustentable de gestión de la calidad educacional planeado en la UCP "José Marti" de Camagüey. En consecuencia, es 
la línea de investigación que identifica al Centro de Estudios para la Evaluación de la Calidad Educacional.

Los resultados obtenidos en estas investigaciones se sintetizan a continuación:

1. La elaboración de una metodología general para la evaluación del impacto social de la formación continua en el contexto y alcance de la institución, la cual toma en cuenta los siguientes fundamentos:

a) La sistematización de contenidos relativos al significado de la calidad de la educación superior pedagógica, asociada a la obtención de la excelencia académica en relación dialéctica con la pertinencia que se refleja en la respuesta al entorno, su interpretación y las posibles acciones de transformación.

b) La evaluación de la calidad implica evaluar la satisfacción ligada a la búsqueda de los impactos sociales. Por tanto, se concreta en que la verdadera calidad es la que tiene un alto impacto social, la que produce transformaciones sociales de envergadura, que se manifiestan mediante niveles de satisfacción en los componentes personal, organizacional y social.

c) La investigación transita desde una macroevaluación de los procesos sustantivos, que se obtiene con la aplicación de un sistema de instrumentos triangulados de acuerdo a las muestras seleccionadas y a los indicadores de cada dimensión que conforman la variable, hasta una microevaluación que se realiza a los resultados generales obtenidos, de acuerdo a los intereses de las personas evaluadoras por encargo del personal que dirige los procesos. Es en este momento en el que se aplican estudios de profundización mediante otra serie de métodos y técnicas, tales como la observación, el análisis, la síntesis, las entrevistas a profundidad semiestructuradas y el muestreo documental, entre otros. 
Bajo estos fundamentos, la metodología general fue aplicada en todas las carreras pedagógicas del territorio, y con los resultados obtenidos se reelaboraron los informes de autoevaluación de las carreras de Educación Especial, Educación Primaria, Educación en Pedagogía, Psicología e Informática, las cuales concluyeron la evaluación y se encuentran en proceso de transformación en aras de alcanzar niveles superiores de calidad educacional, para luego enfrentar en mejores condiciones (preparación del capital humano, transformación del objeto evaluable y optimización de los recursos materiales y financieros) la reevaluación del impacto social y otras evaluaciones de carácter externo que la certifiquen.

2. Otro resultado está relacionado con la evaluación del impacto social de programas de formación académica y la implementación de la autoevaluación de un programa de formación doctoral. Para este último se elaboró un procedimiento de autoevaluación contextualizado, o sea, concertado con la propia formación doctoral y su significado en el contexto y alcance del territorio de Camagüey, correlacionado con las propias bases y preceptos de la metodología general.

\section{Estos programas son:}

- Maestría en Educación Ambiental

- Maestría en Ciencias de la Educación en Red

- Maestría en Educación

- Programa de Formación Doctoral en Ciencias Pedagógicas por la vía tutelar

3. Constituye un resultado significativo la aplicación de operativos de evaluación del impacto social en las actividades de superación realizadas para los y las docentes del subsistema educacional de secundaria básica, donde lo esencial ha sido guiar a los decisores para planificar acciones en el plan de mejoras y, de esta forma, garantizar la excelencia académica en niveles superiores de aceptación social. 
4. Las evaluaciones realizadas dan la posibilidad de elevar el nivel cultural de los y las profesionales de la educación en cuanto a la evaluación del impacto social. Es así que a través del postgrado se continúa potenciando la socialización de los saberes que atesora la universidad y su centro de estudios con respecto a la gestión de la calidad, para lo cual se imparten cursos diseñados e incorporados a la estrategia de superación mediata e inmediata, los cuales se relacionan con las siguientes temáticas:

- Teoría y práctica de la evaluación de la calidad educativa y de su impacto social

- Cultura económica y gestión de la calidad de la educación

- Gestión de información

- Mejoramiento de la Calidad Educativa (MECE)

- Socializar una metodología para la evaluación del proceso docenteeducativo de los y las estudiantes de las carreras pedagógicas

- Perfeccionar la preparación de docentes, de directivos y directivas y de reservas mediante la adquisición de conocimientos fundamentales para el fomento de la cultura económica y la gestión de la calidad de la educación

5. Como regla, a partir de los resultados y del prestigio de la actividad de evaluación del impacto alcanzado por las investigaciones realizadas en el centro de estudios, se contextualiza la metodología general para la evaluación del impacto social en diplomados de diferentes áreas del desarrollo y resultados claves de la universidad, entre ellos destacan:

- Diplomado para Reservas Inmediatas de Directores (DRID)

- Diplomado: Bases Teórico-metodológicas de la Educación Artística desde un Enfoque Humanista y Martiano. Edición II

- Diplomado para perfeccionar la preparación de la reserva educacional prevista para ocupar el cargo de directores y directoras de la escuela contemporánea cubana 
- Diplomado para la preparación de los jefes y las jefas de proyectos para garantizar la dirección efectiva de la actividad científica, con énfasis en la concepción de los proyectos a ciclo completo

6. Un segmento importante de la actividad investigativa corresponde a los encargos y las colaboraciones con otras instituciones de la ciencia en el área de la pedagogía, al igual que con instancias gubernamentales a nivel nacional y territorial.

\section{Conclusiones}

Los resultados del periodo 2012-2014 y la experiencia acumulada por más de una década son una muestra del desempeño de la población docente y las personas investigadoras asociadas a la formación pedagógica en Camagüey en materia de evaluación, con vista a agenciar niveles superiores de calidad educacional y, con ello, de excelencia académica.

Por ello se considera que en la actualidad existe un mayor conocimiento del Sistema de Evaluación y Acreditación para todos los procesos de formación pedagógica que se gestan en el territorio, los cuales se desarrollan con la participación de estudiantes, docentes y directivos y directivas en todas las etapas del proceso de evaluación de la calidad, a partir del conocimiento de los patrones existentes, la sistematización de las experiencias acumuladas, la ejecución de proyectos de investigación sobre evaluación y acreditación de la educación superior pedagógica y del sistema de gestión de la calidad.

Del mismo modo, las investigaciones realizadas en cuanto a la evaluación del impacto social en la UCP "José Martí", además de crear una cultura de gestión sustentable de la calidad, promueven la transformación de la persona y del proceso que se evalúa en relación con la excelencia académica y la pertinencia 
social vista en el entorno, y de acuerdo a la política programática establecida para la educación superior.

\section{Referencias}

Addine, F. y García, G. (2002). La evaluación y acreditación de la educación superior pedagógica. Necesidad y retos para las UCP. La Habana, Cuba: Editorial Pueblo y Educación.

Espí, N. (2012). La Reforma Universitaria y la calidad de la Educación Superior Cubana. CD. Memorias del 8vo Congreso internacional de Educación Superior. "Desarrollo Sostenible". Universidad 2012. La Habana.

Martínez, E. y López, J. (2014). Diversidad en la evaluación del impacto social en la UCP "José Marti". Recuperado de http://www.ecured.cu

López, A. y García, M. (2011). Procedimiento metodológico para la elaboración de plan de mejoras. La Habana, Cuba: Editorial Pueblo y Educación.

Martínez, E., Pozas, W. J., López, J. y Marrero, O. (2013). Metodología para la evaluación del impacto social de la formación continua en la Universidad de Ciencias Pedagógicas "José Marti” Camagüey, Cuba: En formato digital.

Ministerio de Educación (MINED) (2008). Objetivos del Ministerio de Educación, en su labor sistemática por mejorar el proceso de formación de docentes. La Habana, Cuba: Editorial Pueblo y Educación.

Rodríguez, G., Gil, J. y García, E. (2002). Metodología de la investigación cualitativa. PROGRAG, Santiago de Cuba. 